\title{
Obstetric Antiphospholipid Syndrome: Lobsters Only? Or Should We Also Look for Selected Red Herrings?
}

In this issue, a provocative editorial by Clark, Spitzer, and Laskin on obstetric antiphospholipid syndrome (APS), "Has the black swan swallowed a red herring?"1 endorses and strengthens the concerns the Obstetric APS Task Force expressed during the 14th International Congress on Antiphospholipid Antibodies ${ }^{2}$. Such worry relates to the excessive enthusiasm displayed by clinicians when diagnosing and treating obstetric APS, and originates from the paucity of data in support of the association of antiphospholipid antibodies (aPL) and obstetric morbidity as well as from the lack of incontrovertible evidence of the benefit of treatment. Indeed, despite the strong evidence for the pathogenic role of $\beta_{2}$-glycoprotein-I ( $\beta_{2}$-GPI) dependent-aPL in vitro and in vivo models, drawing meaningful conclusions about the clinical significance of aPL is undoubtedly prevented by the several limitations marring available studies. The strength of association is particularly weak when considering aPL and recurrent early miscarriages (REM), as emerged in the critical revision of literature conducted by the international network APS ACTION ${ }^{3,4}$. The concerns over such scarcely supported fervor therefore are not without basis but may go too far, especially when the exclusion of REM from the classification criteria for APS is advocated. In support of such a proposal, the Toronto group claims that aPL-positive women experiencing REM present a favorable outcome regardless of therapeutic intervention, quoting a 2010 study by Cohn, et al. This work does suggest that combining low-dose aspirin [acetylsalicylic acid (LDASA)] plus heparin does not confer any improvement in obstetric outcome among patients with prior REM, but Clark, et al omit that an exception was identified, which concerned women carrying aPL ${ }^{5}$.

Available data about the efficacy of treatment in aPL-positive REM are not univocal, even though all 4 metaanalyses concluded that unfractioned heparin plus LDASA significantly improved obstetric outcome, whereas little evidence emerged for low molecular weight heparin $(\mathrm{LMWH})^{2,6,7}$. Interestingly, the lack of effect by LMWH could be ascribed to the very same Hep/ASA trial mentioned in the editorial. In this 2009 study by Laskin, results might have been biased by inclusion criteria comprising women with REM in the context of positivity for antinuclear antibodies $^{8}$. As a whole, the available burden of evidence surely does not convey a clear-cut demonstration of treatment inefficacy in aPL-positive women with REM. In addition, everyday clinical practice suggests its effectiveness: In a recent survey, $70 \%$ of experts devoted to APS prescribe LDASA plus LMWH not only to patients fulfilling criteria for obstetric APS but also to aPL-positive women with a history of a single or 2 early miscarriages ${ }^{2}$. In support of such an approach, it might be argued that women with a first unexplained early loss and aPL display a higher risk of various pregnancy complications (not only pregnancy loss, but also intrauterine growth restriction, preeclampsia, and abruptio placentae) in a subsequent pregnancy compared to aPL-negative control women ${ }^{9}$.

The Canadian group also stresses the poor predictive ability of isolated positivity for anticardiolipin (aCL) and/or anti- $\beta_{2}$-GPI antibodies as compared to lupus anticoagulant (LAC). We fully agree on the importance of the functional assay as the strongest predictor of pregnancy morbidity; nevertheless, the role of solid-phase criteria aPL tests should not be overlooked. In a UK series of 193 consecutive patients, a single instance of aCL/anti- $\beta_{2}$-GPI antibody positivity was detected in $11.5 \%$ of patients with obstetric APS ${ }^{10}$; in a multicenter European cohort, 31 out of 73 women with purely obstetric APS carried a single positivity in either aCL or anti- $\beta_{2}$-GPI antibody assays ${ }^{11}$. Consistently, double aPL positivity with negative LAC emerged as a strong risk factor for obstetric, but not thrombotic, APS complications, with an OR of $10.8^{12}$. Thus the importance emerges of taking results in ELISA tests into account when diagnosing obstetric APS, even in the context of a mere single positivity at medium-high titers. Further, aPL positivity is increasingly recognized as being associated with pregnancy morbidity, even at low titer. In the above-cited London cohort, over $50 \%$ of women with pure obstetric APS had low-titer aCL and/or anti- $\beta_{2}$-GPI antibodies without $\mathrm{LAC}^{9}$. In a series of 90 Italian subjects with APS, $73.7 \%$ of women with pure obstetric APS had aCL levels between the 99th percentile and 40 IgG phospholipid units, a significantly higher rate compared to

See Obstetric APS: has the black swan swallowed a red herring?,page 155, and Prevalence and significance of aPL, page 210 
patients with thrombotic APS (16.9\%) and those with both conditions $(16.7 \%)^{13}$. Consistently, pregnancy outcomes in untreated patients with low aPL levels and APS-like obstetrical events are similar to women with classical APS; moreover, conventional treatment with LDASA with or without LMWH improved pregnancy outcomes to an extent comparable to otherwise healthy women with REM $^{14}$. Clearly, the whole picture of obstetric APS is multifaceted, and the clinical significance of low-titer aPL positivity in the context of APS pregnancy morbidity is still a matter of debate: participants in the Obstetric Task Force could not agree on a univocal recommendation on whether to treat these women ${ }^{2}$.

Despite the clinical attention reserved to low-titer aPL positivity, we fully agree with Clark, et al about women carrying high-titer aPL experiencing a worse obstetric outcome compared to those with low-titer antibody positivity. However, we still believe that obstetric APS does represent a distinct biological entity in comparison with the vascular variant. For example, $\beta_{2}$-GPI is present only on the endothelial cells of uterine vessels but not on other vascular districts in resting animals; and aPL-positive IgG fractions from pure obstetric patients trigger different cell signaling in comparison with those from thrombotic APS sera ${ }^{15,16}$.

As also stated by Clark, et al, our clinical experience suggests that aPL patients cluster in 2 groups: women with low-risk aPL profile and REM on one side, and those with high-risk aPL profile who experience more severe pregnancy complications such as late losses, preeclampsia, eclampsia, or HELLP syndrome (hemolysis, elevated liver enzymes, and low platelet count) on the other ${ }^{17}$. This clinical observation has recently been confirmed by several lines of evidence. Women with highly positive aPL have been shown to carry an increased risk for adverse fetal and maternal complications: a good pregnancy outcome was registered in only 35\% of pregnancies in women with high-titer aPL, as compared to a 77\% success rate in women with low-titer antibody positivity ${ }^{18}$. Consistently, patients at highest risk - those with triple aPL positivity and a history of thrombosis - have been reported to benefit from additional therapies in terms of pregnancy outcome: a $100 \%$ live birthrate was achieved when patients were treated with LDASA, full-dose LMWH, intravenous immunoglobulin, and low-dose steroids ${ }^{19}$. Most recently, the NOH-APS observational study showed that the obstetric outcome in women with APS receiving treatment depends upon the presenting manifestation: Women with prior late events are at higher risk for an adverse late outcome in the subsequent pregnancy than those with REM ${ }^{20}$. It can thus be envisaged that the clinical presentation might affect the therapeutic efficacy of treatment evaluated in obstetric APS: the standard regimen including LMWH and LDASA might be effective in low-titer aPL positive REM, but not effective enough in late events associated with high-titer aPL. In the latter case, multiple treatments acting on the different
aPL-mediated pathogenic mechanisms could soon become the first-line therapeutic approach.

The emerging interplay between presenting pregnancy complications and response to treatment further highlights the urgent need to better define therapeutic options according to the aPL profile and clinical subset; indeed, we reckon that a large multicenter study is strongly warranted. However, in our opinion, a truly useful study should include the whole spectrum of patients: women with low-titer aPL and those with high-titers, women with single aPL positivity, and those with double/triple aPL positivity, women with REM, and those with late and severe pregnancy complications such as preeclampsia, eclampsia, and HELLP syndrome. The definition of each clinical outcome should be stringent; causes of pregnancy morbidity other than aPL should be carefully excluded; a large (it is hoped) sample size should allow assessing for the first time each outcome individually. This study should also envisage testing for antibodies against domain I of $\beta_{2}$-GPI, phosphatidylserine/prothrombin complex, and phosphatidylethanolamine: These novel diagnostic and prognostic tools should lead to more accurate serological risk stratification ${ }^{21}$.

While conclusive data are awaited, it might be too soon to advocate the exclusion of women with REM from APS classification criteria. REM is not a severe life-threatening condition but surely provides an emotionally painful experience for a woman: In a context where little is known, the border between undertreating and overtreating can be subtle. However, we believe that the risk/benefit ratio favors the treatment option, because the likelihood of therapeutic success is high, with minimal side effects and low costs. In other words, we find it preferable to run the risk of "fishing" for patients who would potentially benefit from treatment options.

\section{PIER LUIGI MERONI, MD; CECILIA B. CHIGHIZOLA, MD,}

Department of Clinical Sciences and Community Health, University of Milan; IRCCS Istituto Auxologico Italiano; MARIA GEROSA, MD;

Department of Clinical Sciences and Community Health, University of Milan; and Istituto G. Pini;

\section{LAURA TRESPIDI, MD; \\ BARBARA ACAIA, MD;}

U.O. Ostetricia e Ginecologia,

IRCCS Fondazione Ca' Granda Ospedale Maggiore Policlinico, Mangiagalli, Regina Elena,

Milan, Italy.

Address correspondence to Prof. Meroni, Istituto Auxologico Italiano, Via Zucchi 18, 20095 Cusano Milanino, Italy.

E-mail: pierluigi.meroni@unimi.it

\section{REFERENCES}

1. Clark CA, Spitzer KA, Laskin CA. Obstetric antiphospholipid syndrome: has the black swan swallowed a red herring? J Rheumatol 2015;42:155-7. Personal non-commercial use only. The Journal of Rheumatology Copyright @ 2015. All rights reserved. 
2. de Jesus GR, Agmon-Levin N, Andrade CA, Andreoli L, Chighizola CB, Porter TF, et al. 14th International Congress on Antiphospholipid Antibodies Task Force report on obstetric antiphospholipid syndrome. Autoimmun Rev 2014;13:795-813.

3. Andreoli L, Chighizola CB, Banzato A, Pons-Estel GJ, de Jesus GR, Erkan D. Estimated frequency of antiphospholipid antibodies in patients with pregnancy morbidity, stroke, myocardial infarction, and deep vein thrombosis: a critical review of the literature. Arthritis Care Res 2013;65:1869-73.

4. Chighizola CB, Andreoli L, Banzato A, de Jesus GR, Pons-Estel GJ, Erkan D. The association between antiphospholipid antibodies and related clinical outcomes: a critical review of the literature [abstract]. Arthritis Rheum 2013;65 Suppl:S1129.

5. Mak A, Cheung MW, Cheak AA, Ho RC. Combination of heparin and aspirin is superior to aspirin alone in enhancing livebirths in patients with recurrent pregnancy loss and positive anti-phospholipid antibodies: a meta-analysis of randomized controlled trials and meta-regression. Rheumatology 2010; 49:281-8.

6. Ziakas PD, Pavlou M, Voulgarelis M. Heparin treatment in antiphospholipid syndrome with recurrent pregnancy loss: a systematic review and meta-analysis. Obstet Gynecol 2010;115:1256-62.

7. Cohn DM, Goddijn M, Middeldorp S, Korevaar JC, Dawood F, Farquharson RG. Recurrent miscarriage and antiphospholipid antibodies: prognosis of subsequent pregnancy. J Thromb Haemost 2010;8:2208-13

8. Laskin CA, Spitzer KA, Clark CA, Crowther MR, Ginsberg JS, Hawker GA, et al. Low molecular weight heparin and aspirin for recurrent pregnancy loss: results from the randomized, controlled HepASA trial. J Rheumatol 2009;36:279-87.

9. Chauleur C, Galanaud JP, Alonso S, Cochery-Nouvellon E, Balducchi JP, Marès $P$, et al. Observational study of pregnant women with a previous spontaneous abortion before 10th gestational week with and without anti-phospholipid antibodies. J Thromb Haemost 2010;8:669-706.

10. Gardiner C, Hills J, Machin SJ, Cohen H. Diagnosis of antiphospholipid syndrome in routine clinical practice. Lupus 2013;22:18-25.

11. Boffa MC, Boinot C, De Carolis S, Rovere-Querini P, Aurousseau $\mathrm{MH}$, Allegri F, et al. Laboratory criteria of the obstetrical antiphospholipid syndrome. Data from a multicentric prospective European women cohort. Thromb Haemost 2009;102:25-8.
12. Pengo V, Biasiolo A, Pegoraro C, Cucchini U, Noventa F, Iliceto S. Antibody profiles for the diagnosis of antiphospholipid syndrome. Thromb Haemost 2005;93:1147-52.

13. Ruffatti A, Olivieri S, Tonello M, Bortolati M, Bison E, Salvan E, et al. Influence of different IgG anticardiolipin antibody cut-off values on antiphospholipid syndrome classification. J Thromb Haemost 2008;6:1693-6.

14. Mekinian A, Loire-Berson P, Nicaise-Roland P, Lachassinne E, Stirnemann J, Boffa MC. Outcomes and treatment of obstetrical antiphospholipid syndrome in women with low antiphospholipid antibody levels. J Reprod Immunol 2012;94:222-6.

15. Agostinis C, Biffi S, Garrovo C, Durigutto P, Lorenzon A, Bek A, et al. In vivo distribution of $\beta 2$ glycoprotein I under various pathophysiologic conditions. Blood 2011;118:4231-8.

16. Lambrianides A, Carroll CJ, Pierangeli SS, Pericleous C, Branch $\mathrm{W}$, Rice J, et al. Effects of polyclonal IgG derived from patients with different clinical types of the antiphospholipid syndrome on monocyte signaling pathways. J Immunol 2010;184:6622-8.

17. Chighizola CB, Gerosa M, Trespidi L, Di Giacomo A, Rossi F, Acaia B, Meroni PL. Update on the current recommendations and outcomes in pregnant women with antiphospholipid syndrome. Expert Rev Clin Immunol 2014;10:1-13.

18. Simchen MJ, Dulitzki M, Rofe G, Shani H, Langevitz P, Schiff E, et al. High positive antibody titers and adverse pregnancy outcome in women with antiphospholipid syndrome. Acta Obstet Gynecol Scand 2011;90:1428-33.

19. Ruffatti A, Salvan E, Del Ross T, Gerosa M, Andreoli L, Maina A, et al. Treatment strategies and pregnancy outcomes in antiphospholipid syndrome patients with thrombosis and triple antiphospholipid positivity. A European multicenter retrospective study. Thromb Haemost 2014;112:727-35.

20. Bouvier S, Cochery-Nouvellon E, Lavigne-Lissalde G, Mercier E, Marchetti T, Balducchi JP, et al. Comparative incidence of pregnancy outcomes in treated obstetric antiphospholipid syndrome: the NOH-APS observational study. Blood 2014;123:404-13.

21. Meroni PL, Chighizola CB, Rovelli F, Gerosa M. Antiphospholipid syndrome in 2014: more clinical manifestations, novel pathogenic players and emerging biomarkers. Arthritis Res Ther 2014;16:209.

J Rheumatol 2015;42:158-60; doi:10.3899/jrheum.141407 